\title{
Zur Ableitung des Braggschen Satzes
}

\author{
Von Georg Menzer \\ Aus dem Kaiser-Wilhelm-Institut für Physik, Max-Planck-Institut, Hechingen \\ (Z. Naturforschg. 2a, 683-684 [1947]; eingegangen am 12. November 1947)
}

Es wird eine vollständige Ableitung des Braggschen Satzes, nach dem die Röntgenstrahlinterferenzen als Reflexe an Netzebenen aufgefaßt werden können, entwickelt.

$\mathrm{U}$ II den Vorgang der Interferenz der Röntgenstrahlen an Kristallgittern zu erklären, kann man sich entweder der Laueschen Gleichungen bedienen oder des Braggschen Satzes, der besagt, daß die gebeugten Strahlen an Netzebenen reflektiert werden, wenn der Spiegelungswinkel $\vartheta / 2$ die Braggsche Gleichung $2 d \sin \vartheta / 2=n \lambda$ erfüllt. Die erste Darstellung wird von Theoretikern, die zweite ihrer Einfachheit und größeren Übersichtlichkeit wegen von Experimentalphysikern, Mineralogen und Chemikern bevorzugt. Bereits 1913 zeigten G. Wulf ${ }^{1}$ für einen speziellen Fall, M. v. Laue ${ }^{2}$ und P. P. Ewald ${ }^{3}$ allgemein, daß die B ragg sche Gleichung sich aus den L a u eschen ableiten läßt. P. P. Ew a ld ${ }^{4}$ wies darauf hin, daß die auf jhr beruhende Erklärung der Beugungserscheinung unvollständig ist; die $\mathrm{zu}$ ihrer Begründung herangezogene Huygenssche Konstruktion der Lichtreflexion trifft nicht für die Beugung der Röntgenstrahlen an einer Netzebene zu, denn von einer Netzebene geht eine größere Anzahl gebeugter Strahlen aus und nicht nur der reflektierte Strahl.

Die Braggsche Darstellung läßt sich jedoch durch eine Ergänzung, wie ich sie mit geringen Abänderungen seit 15 Jahren in meinen Vorlesungen bringe, von der ihr zum Vorwurf gemachten Unvollständigkeit befreien. Für den folgenden Beweis seien als bekannt vorausgesetzt die Sätze:

1. Parallele Strahlen erlangen durch Spiegelung an einer Ebene keine Gangunterschiede gegeneinander.

2. Bei der Spiegelung an einer großen Anzahl von äquidistanten Ebenen, deren gegenseitiger $\mathrm{Ab}$ stand $d$ ist, wird nur dann Licht reflektiert, wenn die Braggsche Gleichung erfüllt ist; aus dieser folgt $d>\frac{1}{2} \lambda$ und $n<\frac{2 d}{\lambda}$.

1 G. Wu lff, Physik. Z. 14, 217 [1913].
3. $n$ ist keine sehr große Zahl (die Erfahrung zeigt, daß $n$ meist 10 bis 20 nicht überschreitet und nur in Ausnahmefällen bei sehr kurzwelliger Strahlung bis zu einigen Hundert ansteigen kann).

Auf ein einfaches Translationsgitter, das längs jeder Gittergeraden eine große Anzahl $N$ von Punkten enthalten soll, falle ein Röntgenstrahl $S$ unter beliebigem Winkel zur Gittergeraden $C_{0}$, deren Identitätsperiode $c$ sei. Von jedem Punkt des Gitters geht, an ihm gestreut, eine Kugelwelle aus. Nun greifen wir eine Richtung $R$ der Kugelwelle heraus. Die Intensität des Strahls $R_{g}$, der sich aus den parallelen Teilstrahlen $R$ zusammensetzt, die von allen Gitterpunkten der Geraden $C_{0}$ ausgehen, ist nur dann von Null verschieden, wenn alle Teilstrahlen $R$ in Phase sind. Legen wir durch jeden Gitterpunkt der Geraden $C_{0}$ eine Hilfsebene $E_{0}$, an der $R$ gegenüber $S$ gespiegelt erscheint, so muß für solche Hilfsebenen die Braggsche Gleichung erfüllt sein, wobei $d=d_{1}$ der Abstand benachbarter Ebenen $E_{0}$ ist.

Gehen wir von $C_{0}$ zu einer benachbarten Gittergeraden $C_{1}$ und $\mathrm{zu}$ den folgenden Gittergeraden $C_{2}, C_{3}, \ldots$ über, die mit $C_{0}$ und $C_{1}$ in einer Ebene liegen, so wird das System der Hilfsebenen $E_{1}$, die parallel $\mathrm{zu} E_{0}$ durch die Gitterpunkte der Geraden $C_{1}$ gelegt seien, im allgemeinen um die Strecke $d_{2} \neq d_{1}$ senkrecht zu $E_{0}$ gegenüber dem System der $E_{0}$ verschoben sein, das System $E_{2}$ um $2 d_{2}$ usw. Der an der gesamten Netzebene gebeugte Strahl $R_{e}$, der sich aus allen von den einzelnen Gittergeraden $C$ herrührenden Strahlen $R_{g}$ zusammensetzt, wird daher nur dann nicht ausgelöscht sein, wenn auch $d_{2}$ ebenso wie $d_{1}$ die Braggsche Gleichung befriedigt.

Der gleiche Schritt wiederholt sich, wenn man von der Netzebene $C_{0}, C_{1}, C_{2}, \ldots$ über die benach-

2 M. L a u e, Physik. Z. 14, 421 [1913].

3 P. P. Ew ald, Physik. Z. 14, 465 [1913].

${ }^{4}$ P. P.Ew al d, Kristalle und Röntgenstr. 1923, S. 51. 
barten Netzebenen $C_{0}^{\prime}, C_{1}^{\prime}, C_{2}^{\prime}, \ldots, C_{0}^{\prime \prime}, C_{1}^{\prime \prime}, G_{2}^{\prime \prime}, \ldots$ zum Raumgitter übergeht. Die entsprechenden Hilfsebenen $E^{\prime}, E^{\prime \prime}, \ldots$ sind gegen die $E$ um $d_{3}$, $2 d_{3}$ usw. verschoben. Die von den einzelnen Netzebenen herrührenden Strahlen $R_{e}, R_{e}^{\prime}, R_{e}^{\prime \prime}, \ldots$ heben sich nur dann nicht auf, wenn auch $d_{3}$ zugleich mit $d_{1}$ und $d_{2}$ die Braggsche Gleichung erfüllt. Das ist nur möglich, wenn

$$
\frac{d_{1}}{n_{1}}=\frac{d_{2}}{n_{2}}=\frac{d_{3}}{n_{3}}=\delta,
$$

worin $n_{1}, n_{2}, n_{3}$ drei ganze Zahlen sind, die wegen des dritten, als bekannt vorausgesetzten Satzes nicht sehr groß sein dürfen. Durch dic Gittergerade $C_{0}$ gehen im Bereich einer Identitätsperiode $c$ folglich $d_{1} / \delta=n_{1}$ Hilfsebenen $E$ und somit rund $N n_{1}$ Hilfsebenen durch die ganze Gittergerade $C_{0}$. Da es im Gitter rund $N^{3}$ Punkte gibt, müssen $N^{3} / N n_{1}=N^{2} / n_{1}$ Punkte auf jeder Hilfs- ebene liegen, also mehr Punkte. als selbst auf einer Gittergeraden Platz haben. Die Hilfsebenen müssen also Netzebenen sein, womit der Braggsche Satz bewiesen ist: ein gebeugter Strahl tritt dann und nur dann auf, wenn er die Richtung des an einer Netzebene gespiegelten Primärstrahls hat und die Braggsche Gleichung für diese Netzebene erfuillt ist. Dann sind auch die von allen Gitterpunkten herrührenden Teilstrahlen $R$ in Phase.

Die hier entwickelte Ableitung des Braggschen Satzes berührt sich stellenweise mit der L a u eschen Darstellung. Aus dem Satz, daß alle gebeugten parallelen Teilstrahlen $R$ in Phase sind, folgen unmittelbar die Laueschen Gleichungen. Es fehlen hier die Interferenzkegel; das ist aber kein Nachteil. Wo man sie braucht, wie etwa bei den Schichtlinien der Drehkristallmethode oder bei Laue-Aufnahmen, lassen sie sich mühelos einführen.

\title{
NOTIZEN
}

\section{Modellbetrachtungen zum Problem der Biradikale}

\author{
Von Hermann Hartmann \\ Institut für physikalische Chemie \\ der Universität Frankfurt a. M.
}

(Z. Naturforschg. 2a, 684 [1947]; eingeg. am 8. Oktober 1947)

Als Biradikale bezeichnet man Moleküle, bei denen der tiefste Singulettzustand und der tiefste Triplettzustand praktisch miteinander entartet sind.

Bisher liegt nur ein Versuch von $\mathrm{H}$ ü $\mathrm{ck} \mathrm{e}{ }^{1}{ }^{1}$ vor, für den Schlenkschen Kohlenwasserstoff die Lage der fraglichen Terme zueinander theoretisch zu bestimmen. Die Hückelsche Rechnung wurde mit Hilfe de,,zweiten“ Näherungsverfahrens ausgeführt. Das dem genannten Problem wesentlich besser angepaßte ,erste“ Näherungsverfahren (nach Slater-Hückel-Pauling) ist bisher nicht angewandt worden.

Wir haben für zwei Modellmoleküle, und zwar das klassisch formulierbare Butadien (I) und das klassisch nicht formulierbare, also in gewissem Sinne ,,metachinoide“ Trimethylenmethyl (II)

$$
\mathrm{CH}_{2}=\mathrm{CH}-\mathrm{CH}=\mathrm{CH}_{2}
$$

$$
\text { I }
$$<smiles>CC(C)C</smiles>

mit dem ersten Näherungsverfahren die tiefsten singulett- und Triplett-Terme berechnet. Die Resultate sind in Abb. 1 dargestellt. $E$ ist die Termenergie, $C$ ein für die relative Lage der Terme belangloses Coulombintegral, $A$ ein (negatives) Austauschintegral, dessen
Betrag etwa $40 \mathrm{kcal} / \mathrm{Mol}$ ist. Unter 1 sind Singulett-, unter 3 Triplett-Terme eingezeichnet.

Wie zu erwarten war, liegt bei dem klassisch formulierbaren Butadien der erste Triplett-Term weit (etwa $30 \mathrm{kcal} / \mathrm{Mol}$ ) über dem Singulettgrundterm. Bei

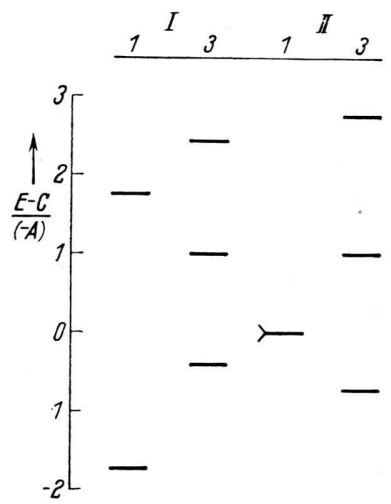

Abb. 1. Termschema von Butadien (I) und Trimethylenmethyl (II).

der metachinoiden Modellsubstanz Trimethylenmethyl ist der Grundterm ein Triplett. Der tiefste Singulett Term liegt jedoch so weit über diesem Grundterm. dak keineswegs ein Biradikal vorliegt.

1 E. $\mathrm{H}$ ückel, Z. Elektrochem. angew. physik. Chem. 43, $834 \mathrm{ff}$. [1937]. Bei einer Untersuchung des m-Benzodimethids durch F. Se el, Z. physik. Chem. Abt. B, 51, 229 [1942], fehlt die Berücksichtigung der Austauschentartung der ..letzten" beiden Elektronen. 